\title{
International Year of the Planet Earth (IYPE) und unsere FH-DGG-Tagung in Göttingen, Mai 2008
}

\author{
Martin Sauter \\ Online veröffentlicht: 22.1.2008 \\ (C) Die Autoren 2008
}

Liebe Leserinnen und Leser, nach dem Beschluss der sogenannten „Milleniumsziele“ durch die Vereinten Nationen, die u.a. die Internationale Dekade „Water for Life, 2005-2015“ initiierten (siehe Leitartikel Grundwasser, Juni 2005), wurde von der UNGeneralversammlung am 22. Dezember 2005 das Jahr 2008 zum Internationalen Jahr des Planeten Erde ausgerufen (International Year of the Planet Earth, IYPE, www.geounion.de). Damit stehen 2008, nach dem vom BMBF im Jahr 2002 veranstalteten nationalen „Jahr des Planeten Erde“, die Geowissenschaften wiederum öffentlichkeitswirksam im Rampenlicht.

Als Hauptziele wurden neben der Minimierung von Georisiken, der Ermittlung der geogenen Prozesse beim

\author{
Martin Sauter \\ Geowissenschaftliches Zentrum \\ der Universität Göttingen \\ Abt. Angewandte Geologie \\ Goldschmidtstrasse 3, 37077 Göttingen \\ E-Mail: martin.sauter@geo.uni-goettingen.de
}

Klimawandel, der Exploration von Ressourcen, der Vermittlung geowissenschaftlicher Zusammenhänge in Gesellschaft und Studium, wiederum die Erschließung von Grundwasser in den Vordergrund gestellt.

Von der Deutschen UNESCO-Kommission wurden zehn verschiedene Schwerpunktforschungsthemen definiert, wobei eines der Themen „Grundwasser: auf dem Wege zu nachhaltiger Nutzung" ist.

Die für den 21. bis 25. Mai 2008 unter dem Motto „Grundwasserressourcen - Charakterisierung, Bewirtschaftung, Prognosen " in Göttingen geplante Tagung der Fachsektion Hydrogeologie erfährt damit sowohl die politische als auch die internationale Unterstützung.

Themen unserer Tagung sind neben den hydrogeologischen Charakteristiken der verschiedenen Locker- und Festgesteinsgrundwasserleiter, insbesondere methodische Aspekte, wie z.B. Charakterisierungs- und Modelltechniken, sowie Verfahren der Grundwasserbewirtschaftung. Begleitet wird die Ta- gung von Fortbildungsveranstaltungen, die den Einsatz hydrogeophysikalischer Methoden zur Charakterisierung von Grundwasserleitern, neueste Entwicklungen in der reaktiven Stofftransportmodellierung und die Quantifizierung von Schadstoffflüssen in Einzugsgebieten zum Thema haben. Als Referenten konnten wir international ausgewiesene Fachleute gewinnen. Ferner sind Exkursionen zu den Harzwasserwerken, in den Karst des Südharzes und zu den hydrogeologischen Besonderheiten der Umgebung Göttingens geplant. Weitere Details finden Sie im GrundwasserHeft 3/2007 (S. 248-251).

Sie sind alle in Göttingen herzlich willkommen,

Ihr Martin Sauter

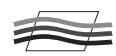

Open Access Dieser Artikel wird zu den Bedingungen der „Creative Commons Attribution Noncommercial License" zur Verfügung gestellt. Damit ist eine nichtkommerzielle Nutzung, Verbreitung und Vervielfältigung erlaubt, sofern die Autoren des Artikels und die genaue Quelle angegeben sind. 